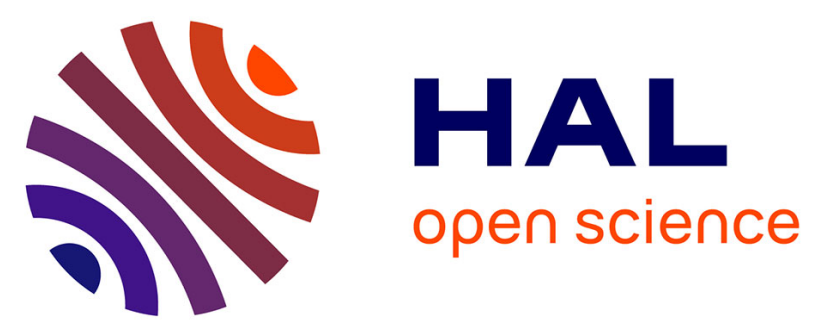

\title{
Hydrodynamique de l'Orne et mobilisation sédimentaire dans la zone de remous amont du barrage de Beth (Lorraine, France)
}

Benoît Losson, Luc Manceau, Hussein Kanbar, Yves Waldvogel, Claire Delus, Laurence Mansuy-Huault, Christophe Hissler, Emmanuelle

Montargès-Pelletier

\section{To cite this version:}

Benoît Losson, Luc Manceau, Hussein Kanbar, Yves Waldvogel, Claire Delus, et al.. Hydrodynamique de l'Orne et mobilisation sédimentaire dans la zone de remous amont du barrage de Beth (Lorraine, France). Géomorphologie: relief, processus, environnement, 2020, 26 (1), 10.4000/geomorphologie.14004 . hal-02615662

\section{HAL Id: hal-02615662 \\ https://hal.science/hal-02615662}

Submitted on 16 Nov 2020

HAL is a multi-disciplinary open access archive for the deposit and dissemination of scientific research documents, whether they are published or not. The documents may come from teaching and research institutions in France or abroad, or from public or private research centers.
L'archive ouverte pluridisciplinaire HAL, est destinée au dépôt et à la diffusion de documents scientifiques de niveau recherche, publiés ou non, émanant des établissements d'enseignement et de recherche français ou étrangers, des laboratoires publics ou privés. 


\section{Hydrodynamique de l'Orne et mobilisation sédimentaire dans la zone de remous amont du barrage de Beth (Lorraine, France)}

(titre court : Hydrodynamique de l'Orne et mobilisation sédimentaire)

\section{Orne River hydrodynamic and effect on sediment transport in the backwater zone of the Beth Dam (Lorraine, France)}

Benoît Losson ${ }^{* a, c}$, Luc Manceau ${ }^{\text {a, c }}$, Hussein J. Kanbar ${ }^{\text {b, c }}$, Yves Waldvogel ${ }^{\text {b, c }}$, Claire Delus ${ }^{\text {a, c }}$, Laurence Mansuy-Huault $^{b, c}$, Christophe Hissler ${ }^{d}$, Emmanuelle Montargès-Pelletier ${ }^{b, c}$

${ }^{a}$ Université de Lorraine, LOTERR (Centre de recherche en géographie), E.A. 7304, U.F.R. S.H.S.Metz, Ile du Saulcy, B.P. 60228, 57045 Metz cedex 01, France.

b C.N.R.S., Université de Lorraine, LIEC (Laboratoire interdisciplinaire des environnements continentaux), U.M.R. 7360, 54500 Vandœuvre-lès-Nancy, France.

${ }^{\mathrm{c}}$ L.T.S.E.R. France, Zone Atelier du Bassin de la Moselle, Nancy, France.

${ }^{\mathrm{d}}$ Luxembourg Institute of Science and Technology (LIST), Environmental Research and Innovation Department, 41 rue du Brill, 4422 Belvaux, Luxembourg.

*Auteur correspondant. Tél : +33 (0)372748344

Courriels :

benoit.losson@univ-lorraine.fr (B. Losson)

luc.manceau@univ-lorraine.fr (L. Manceau)

Hsen.kanbar@gmail.com (H. J. Kanbar)

yves.waldvogel@univ-lorraine.fr(Y.Waldvogel)

claire.delus@univ-lorraine.fr (C. Delus)

laurence.mansuy@univ-lorraine.fr (L. Mansuy-Huault)

christophe.hissler@list.lu (C. Hissler)

emmanuelle.montarges@univ-lorraine.fr (E. Montargès-Pelletier)

\section{Résumé}


L'Orne a subi de multiples modifications hydromorphologiques au cours du 20e siècle, en relation avec l'activité sidérurgique implantée dans sa vallée aval. A Moyeuvre-Grande, le barrage de Beth, construit en 1959, provoque notamment une zone de remous de 4,5 km de longueur, présentant localement des bancs alluviaux ainsi que des dépôts solides fortement contaminés (métaux, hydrocarbures aromatiques polycycliques, ...) directement liés aux industries. Une étude en cours vise à déterminer la remobilisation de ces dépôts pollués dans les conditions hydrologiques actuelles et dans une perspective d'effacement du barrage.

Le travail présenté ci-après concerne spécifiquement les caractéristiques hydrodynamiques de l'Orne dans la zone de ralentissement provoqué par le barrage. La crue biennale du $1^{\text {er }}$ juin 2016 est utilisée comme événement de référence ; son débit maximal à $137 \mathrm{~m}^{3} / \mathrm{s}$ correspond localement au débit à pleins bords. Deux sites avec banc sédimentaire, éloignés de $450 \mathrm{~m}$, ont fait l'objet de mesures morphométriques permettant de calculer la puissance spécifique et la contrainte tractrice sur des tronçons de 37 et $59 \mathrm{~m}$ de long respectivement. Sur les bancs, des dalles ont été mises en place, pour mesurer les taux de sédimentation, récupérer les sédiments et les analyser d'un point de vue granulométrique.

La puissance spécifique (environ $30 \mathrm{~W} / \mathrm{m}^{2}$ ) et les contraintes tractrices moyennes (entre 15 et $22 \mathrm{~N} / \mathrm{m}^{2}$ ) calculées, révèlent un cours d'eau d'assez faible énergie hydrodynamique, qui peut toutefois mobiliser des sédiments de taille centimétrique par charriage et du sable moyen à grossier en suspension, lors d'une crue biennale.

Ces premiers résultats constituent un point d'appui pour une future modélisation hydraulique à mener à proximité du barrage, afin de déterminer les processus d'ablation qui pourraient s'y produire, notamment lors de l'effacement annoncé de l'ouvrage.

Mots-clés : puissance spécifique, contrainte tractrice, barrage, remous, contamination industrielle, Lorraine, Orne.

\section{Abstract}

Hydromorphological changes were applied to the Orne River during the $20^{\text {th }}$ century to suit the demands of the steel industries. In the downstream region of the Orne valley, near MoyeuvreGrande, the Beth Dam was constructed in 1959. This dam causes a $4.5 \mathrm{~km}$ long backwater zone, 
which locally displays sedimentary deposits due to the flow decrease as highly contaminated waste deposits (metals, polycyclic aromatic hydrocarbons, etc.) directly linked to the ancient steel making activity. An ongoing study aims to determine the remobilization of these polluted deposits in the current hydrological conditions. Consequently, improved management plans can be proposed before the dam will be removed.

This article specifically focuses on the hydrodynamic characteristics of the Orne River in the upstream slowing zone, caused by the Beth Dam. The biennial flood of June $1^{\text {st }} 2016$ serves as a reference; its maximum flow rate of $137 \mathrm{~m}^{3} / \mathrm{s}$ corresponds to the bankfull discharge. Morphometric measurements were done on river bars of two sites (450 $\mathrm{m}$ apart). Subsequently, the unit stream power and the shear stress were calculated on sections of 37 and $59 \mathrm{~m}$ length. Slabs were put on the bars to measure sedimentation rates, to collect sediments, and to analyze their grain size.

The calculated unit stream power (about $30 \mathrm{~W} / \mathrm{m}^{2}$ ) and mean shear stresses (between 15 and $22 \mathrm{~N} / \mathrm{m}^{2}$ ) reveal a stream of rather low hydrodynamic energy. Nonetheless, it is more than enough to mobilize centimetric sediments via bed load transport and medium to coarse sands via suspension during a biennial flood.

These results are a starting point for future hydraulic modelling that will be carried out near the Beth Dam in order to determine the possible ablation processes that will occur in case of dam removal.

Key words: unit stream power, shear stress, dam, backwater, industrial contamination, Lorraine, Orne.

\section{Abridged English Version}

The Orne valley, located in northern Lorraine (north-east of France), witnessed intense industrial activities at the end of the $19^{\text {th }}$ century and in the $20^{\text {th }}$ century, mainly associated with the exploitation of iron ore (Garcier, 2007). The installation of steelworks at the riverbanks had been accompanied by multiple hydromorphological changes (fig. 1). At Moyeuvre-Grande in particular, a dam with gates was built in 1959 (Beth Dam; fig. 2A), causing a backwater zone of over $4.5 \mathrm{~km}$ in length. Although a big part of the riverbed in the upstream vicinity of the Beth Dam was dredged in the 1960s and 1980s, some sections of the Orne River still display highly contaminated sedimentary deposits (metals, polycyclic aromatic hydrocarbons, etc.). Indeed, under a few centimeters of lithogenic sedimentary materials lie metal enriched deposits that resulted from past mining and 
metallurgical activities (Kanbar, 2017; Kanbar et al., 2017). The current study, entitled MOBISED ("Modelling the remobilization of sediments and the release of the associated contaminants »), aims to determine the resuspension possibility of these polluted sediments in the present hydrological conditions, especially since the dam is planned to be removed in the near future (perhaps in 2020). In this context, hydrodynamic measurements and calculations have been developed to identify the sediment transport capacities during high flows.

In this paper, we focus on the hydrodynamic characteristics of the Orne River in the slowing zone of the current, i.e. in the upstream area of the backwater caused by the Beth Dam. The biennial flood that occurred on June $1^{\text {st }} 2016$ is examined; it had a maximum flow of $140 \mathrm{~m}^{3} / \mathrm{s}$. A measurement of about $137 \mathrm{~m}^{3} / \mathrm{s}$ was made at that time at Haropré footbridge (fig. 3A, 3D and 4) just after the flood peak. This value, which corresponds to the bankfull discharge, was used for the hydraulic computations. A steady slope of the water level of $0.0915 \%$ over the entire length was considered (fig. 3E). Two sites, $450 \mathrm{~m}$ apart, are compared in this study, namely Haropré (HP5) and Sainte-Anne (SA1); both sites have a bar located on the right bank (fig. 2B, 3B and 3C). They were the subject of precise morphometric measurements to calculate the unit stream power and the mean shear stress on sections of 37 and $59 \mathrm{~m}$ long. On each of the bars, slabs were laid to measure sedimentation rates, to collect sediments, and to analyze them from a grain-size and geochemical viewpoints; we rely on a sand bed collected on slab SA1-04be (fig. 5A and 5B) and a sand unit sampled by coring (JOSAN15C3). Moreover, sediment samplings in the bedload of the Orne River were made in the immediate vicinity of the two bars (HP5-1 and SA1-1; fig. 3B and 3C).

The two study sites showed comparable unit stream power values ( $30 \mathrm{~W} / \mathrm{m}^{2}$; fig. 6). This indicates that the Orne is hardly capable of morphological changes in the backwater zone due to its weak hydrodynamic energy. However, the flow velocities at HP5 and SA1 (with 1.15 and $1.39 \mathrm{~m} / \mathrm{s}$ averages, respectively) allow ablation and transport of centimeter-sized sediments during a biennial flood (see Hjulström diagram; Chamley, 2000).

The mean shear stresses at HP5 and SA1 are about 15 and $22 \mathrm{~N} / \mathrm{m}^{2}$, respectively (fig. 6). Based on numerous studies on the relationship between critical shear stress and grain-size distribution (Bravard and Petit, 2002), our data make it possible to establish a minimum size range of particles that are remobilized by the flow during a flood (fig. 7A). Grain sizes range from 15 to $81 \mathrm{~mm}$ and 22 to $99 \mathrm{~mm}$ at HP5 and SA1, respectively. These results can be completed and specified by a second approach related to the Shields parameter.

The median particle size (D50) of the two samples HP5-1 and SA1-1, gave Shields parameter values of 0.39 and 0.27, respectively (fig. 8). These values are well above the bedload transport thresholds proposed by A. Shields and by M. Ramette ( $\tau^{*}$ between 0.047 and 0.06; see Degoutte, 
2006). For $\tau^{*}>0.047$, in a non-paved gravel river context, the shear stress values provide a Shields parameter that expresses an initiation of bedload motion for particles up to $19.9 \mathrm{~mm}$ in diameter at HP5 and $28.7 \mathrm{~mm}$ at SA1. This indicates that $90 \%$ and $99 \%$ of the riverbed particles at HP5 and $S A 1$, respectively, could remobilize during the flood (fig. $7 B$ ).

Suspended transport of sands smaller than $370 \mu \mathrm{m}$ at HP5 and $540 \mu \mathrm{m}$ at SA1 could occur during the flood peak (according to the classification of M. Ramette). According to R.A. Bagnold, particles up to $1.1 \mathrm{~mm}$ might resuspend (tab. 1). On the other hand, the results based on the threshold of L.C. Van Rijn are significantly different from the sedimentary data obtained on SA1 bar (fig. 9); this goes for both the sedimentation slab (SA1-04be; deposit of 2016 with a coarse percentile C1 of $941 \mu \mathrm{m})$ as well as the core (JOSAN15C3-29; sand unit of a probable post-1967 deposit, even 1988, with a coarse percentile C1 of $758 \mu \mathrm{m}$ ). The slab SA1-04be, in particular, presents mainly fine and medium sands (fig. 5B and 9: median at $200 \mu \mathrm{m}$, mean size of Folk and Ward at $130 \mu \mathrm{m}$; see Miskovsky and Debard, 2002). This is confirmed by observations of superficial deposits of fine sand on riparian vegetation after the flood and by the absence of gravel particles on any sedimentation slab on the bar surfaces or in the cored sediments.

The floods of the Orne River are therefore well able to remobilize the entire range of silty to gravelly particles in the study area. The geochemical, mineral and organic analyzes of the sandyloam sediments of Sainte-Anne's bar revealed relatively low contamination compared to other deposits further downstream in the backwater zone of the Beth Dam. The grain-size characteristics are significantly lower near the dam (average size around $14 \mu \mathrm{m}$, against $37 \mu \mathrm{m}$ in Sainte-Anne) and with a strongly cohesive character. Nonetheless, coarse sand deposits also exist at the bottom of the riverbed (fig. 2A). The measurements made are considered an important step in determining the ablation processes that could be involved downstream if the dam is removed.

\section{Introduction}

Historiquement, les cours d'eau ont d'abord été considérés comme des ressources pour les populations, en termes de subsistance (eau, faune et flore aquatiques) ou de développement économique (voie de communication, énergie hydraulique). Leurs potentialités agricoles et industrielles ont généré des aménagements multiples, parfois outranciers, tout comme certains besoins de maîtrise des contraintes hydrologiques (crues et étiages). Ce n'est qu'avec l'apparition de désagréments pour les sociétés, qu'une nouvelle perception des milieux aquatiques a pu naître, par exemple en France avec la Loi sur l'eau de 1992 et en Europe avec l'élaboration de la Directive 
cadre sur l'eau en 2000. L'intégration des cours d'eau dans les écosystèmes des zones humides s'accompagne de politiques de gestion davantage tournées vers la qualité des milieux fluviaux et de leur fonctionnement.

Dans ce cadre, des organismes tels que les agences de l'eau ou des établissements publics de coopération intercommunale désormais compétente en GEMAPI (Gestion des milieux aquatiques et prévention des inondations), mettent en œuvre des programmes de protection de sites, d'entretien d'ouvrages hydrauliques ou visant le bon état chimique et écologique des masses d'eau. L'une de leurs problématiques concerne alors le devenir des seuils et barrages qui n'ont plus d'utilité : faut-il les supprimer, les araser ou les réaménager?

Cette thématique de la gestion des anciens ouvrages recoupe la question du fonctionnement physique des cours d'eau, à travers l'hydrodynamique, la morphologie fluviale et le transport sédimentaire. De nombreuses études ont déjà examiné les conséquences futures ou effectives de l'effacement de seuils (par exemple Syed et al., 2005 ; Cheng et Granata, 2007 ; Melun, 2012). Nous nous plaçons dans une démarche prospective visant à déterminer la mobilisation des sédiments stockés dans la zone de remous d'un petit barrage implanté au fil de l'eau sur le cours de l'Orne, en Lorraine. L'étude globale, intitulée MOBISED («Modelling the remobilization of sediments and the release of the associated contaminants ») et soutenue par l'Agence Nationale de la Recherche (ANR-14-CE01-0019), s'intéresse à la remise en suspension des particules et de leurs contaminants, hérités de l'activité industrielle qui a jouxté la rivière pendant un siècle. Cependant, dans cet article, nous nous limitons thématiquement à l'identification des paramètres hydrodynamiques de mise en mouvement des sédiments (puissance spécifique et contrainte tractrice) et géographiquement à la partie amont de la zone de remous engendrée par le barrage.

\section{Contexte et objectif de l'étude}

Située dans le nord-est de la France, la vallée de l'Orne a connu une intense activité industrielle dès la fin du 19e siècle, associée à l'exploitation industrielle du minerai de fer lorrain (Garcier, 2007). L'installation des usines minières et sidérurgiques au bord du cours d'eau s'est accompagnée de multiples modifications hydromorphologiques (fig. 1) : déplacement du lit mineur et corsetage de la rivière sur certains tronçons, enrochements massifs de berges, implantation d'ouvrages transversaux, rejets solides volumineux (Martinois, 2014). Le démantèlement des établissements industriels liés à l'acier, à partir des années 1980, a libéré une partie de l'ancien lit majeur de l'Orne, bien que certaines friches aient été réemployées par de nouvelles activités à vocation 
commerciale ou industrielle (Picon, 2014). Le lit mineur a subi ponctuellement quelques travaux de requalification, notamment en lien avec les aménagements du " Fil bleu ", promenade courant le long de la rivière (E.P.F.L., 2009). Cependant, il n'y a pas encore eu à ce jour de restauration de fond du milieu fluvial, seulement des diagnostics et scénarii de réaménagement des infrastructures barrant le cours d'eau (SINBIO, 2013a, 2013b). Ainsi, à Moyeuvre-Grande en particulier, le barrage à vannes levantes de Beth, de $4 \mathrm{~m}$ de haut (fig. 2A), provoque une zone de remous de plus de 4,5 km de longueur (fig. 1) ; cet ouvrage construit en 1959, avait pour vocation de constituer une réserve d'eau, notamment pour le refroidissement des hauts-fourneaux des Forges de Jœuf, établis en amont immédiat du barrage au tournant des années 1950-60.

Il est bien établi que les zones de remous sont favorables à la sédimentation des particules, par ralentissement du flux d'eau, théoriquement sous forme de sédiments grossiers à l'amont et plus fins vers l'aval (Degoutte, 2006 ; Manière, 2013). Bien que curé en 1967 à l'amont du remous et en 1988 dans la zone de remous (Martinois, 2014), ce tronçon de l'Orne présente de manière éparse des bancs plus ou moins sableux ou vaseux, de manière discontinue, sur tout son linéaire. A l'aval de Jœuf, à proximité des anciens sites sidérurgiques et jusqu'au barrage, des dépôts fortement contaminés (métaux, hydrocarbures aromatiques polycycliques, etc.), empreints de l'activité métallurgique, à peine recouverts par quelques centimètres de matériaux d'origine plus naturelle, ont été décelés et analysés (Kanbar, 2017 ; Kanbar et al., 2017). Dans la partie amont de la zone de remous, des bancs non contaminés, constitués essentiellement de composants naturels, donc postcurage 1967, se sont vraisemblablement formés en quelques dizaines d'années, sous l'influence de différents facteurs naturels (géométrie et morphologie du chenal, végétation rivulaire), associés à la décélération des écoulements (fig. 2B).

L'étude menée depuis 2014 vise à déterminer la remise en suspension des sédiments dans les conditions hydrologiques actuelles et dans une perspective d'effacement du barrage de Beth, qui pourrait avoir lieu en 2020. Dans ce cadre, des mesures et calculs hydrodynamiques ont été développés, pour identifier les capacités de transport sédimentaire de la rivière pendant les phases d'intumescence hydrologique. C'est ce volet hydrosédimentaire que nous proposons d'exposer dans les lignes qui suivent, en se focalisant sur la zone amont du remous provoqué par le barrage (fig. 1). Le choix de ce secteur a été dicté par plusieurs conditions : la présence de bancs de sédiments fins partiellement et temporellement immergés, un contexte hydromorphologique permettant de réaliser des mesures topographiques, des expériences de sédimentation et des prélèvements relativement aisés (compte tenu de la tranche d'eau limitée), la possibilité d'effectuer des mesures hydrologiques en phase de crue grâce à l'existence d'une passerelle. On ajoutera qu'en période de crue, l'entrée dans la zone de remous est caractérisée par un comportement hydrodynamique de l'Orne proche de 
son cours naturel, donc avec une problématique de mobilisation et de transit sédimentaire qui pourra être appliquée sur l'ensemble de la zone d'influence du barrage en cas d'arasement ou de suppression totale.

\section{Méthodologie : les mesures hydrologiques, topographiques et sédimentologiques à l'appui des calculs hydrodynamiques}

Des stations hydrométriques implantées à l'amont du secteur d'étude (Jœuf Abattoir - JOAB) et au barrage de Beth (fig. 1), enregistrent le niveau de l'Orne en continu depuis décembre 2014, respectivement par sonde de pression Orpheus Mini OTT et capteur bulle à bulle OTT. Des campagnes régulières de mesure de débit à la perche de jaugeage à intégration ou à l'A.D.C.P. (mesureur de vitesse à effet Doppler) Streampro Teledyne RD, permettent d'obtenir un hydrogramme complet sur plus de 4 ans. Les deux stations fournissent quasiment les mêmes valeurs, puisque l'Orne ne reçoit aucun affluent dans l'intervalle de distance, uniquement une petite source, du ruissellement et des rejets de la station d'épuration de Jœuf. Deux stations hydrométriques de la DREAL Lorraine situées à Moyeuvre-Grande, $750 \mathrm{~m}$ en aval du barrage de Beth, et à Rosselange, environ 3,5 km en aval du barrage et à l'aval du confluent du Conroy et d'exhaures minières, permettent également de replacer les mesures obtenues dans un contexte hydrologique plus long (stations en activité depuis 2003 et 1967 respectivement ; Banque HYDRO, 2019a, 2019b). Les principaux biais hydrologiques qui peuvent intervenir et qui ont dû nécessiter un traitement des données, sont liés aux manœuvres fréquentes des vannes du barrage de Beth, mais également aux interventions anthropiques du même type qui ont lieu régulièrement sur le barrage d'Homécourt, situé en amont du tronçon étudié (fig. 1).

Sur la période de mesure, le débit journalier maximum de l'Orne a dépassé $160 \mathrm{~m}^{3} / \mathrm{s}$ à la station $\mathrm{JOAB}$, le 4 juin 2016 ; cette crue est aussi la plus importante enregistrée à la station de MoyeuvreGrande depuis son implantation (Banque HYDRO, 2019a). Cet événement a été précédé par trois autres pics de crues (fig. 3D), notamment le $1^{\text {er }}$ juin où le débit moyen journalier a presque atteint les $130 \mathrm{~m}^{3} / \mathrm{s}$. Cette crue du $1^{\text {er }}$ juin 2016 est considérée comme biennale sur une période de 39 ans à la station de Rosselange et quinquennale par les calculs sur 12 ans à la station de Moyeuvre-Grande (Banque HYDRO, 2019a, 2019b). Une mesure instantanée à près de $137 \mathrm{~m} / \mathrm{s}$ a été réalisée à cette date à la passerelle d'Haropré (fig. $3 \mathrm{~A}$ et 4), juste après le pic de crue. C'est cette valeur, accompagnée de mesures morphométriques du chenal, que l'on a retenue pour les calculs 
hydrauliques qui seront présentés et effectués ci-après ; elle correspond localement au débit à pleins bords.

Deux sites (Haropré - HP5 et Sainte-Anne - SA1), éloignés d'environ $450 \mathrm{~m}$ et localisés de part et d'autre de la passerelle où la mesure de débit a été obtenue, sont comparés (fig. 3A) ; l'un comme l'autre sont constitués d'un banc sédimentaire en rive droite. Ils ont fait l'objet de mesures morphométriques précises au tachéomètre Geodimeter et au GPS différentiel monofréquence Trimble 4600LS (Perrot, 2014 ; Justica, 2015), permettant d'obtenir des topographies des secteurs sélectionnés (fig. 3B et 3C) et de calculer ensuite la puissance spécifique et la contrainte tractrice globale sur des tronçons de 37 et $59 \mathrm{~m}$ de long respectivement. Lors de la crue du $1^{\text {er }}$ juin 2016, des repères de niveau d'eau ont été matérialisés sur chacun des sites et mesurés ultérieurement.

Enfin, sur les bancs des deux sites, des dalles ont été mises en place depuis septembre 2015, afin de mesurer les taux de sédimentation, récupérer les sédiments et les analyser d'un point de vue granulométrique et géochimique (fig. 5A et 5B). Au nombre de 14 sur les deux bancs la première année, certaines dalles étaient relevées après chaque crue dans la mesure des possibilités d'accès, d'autres annuellement. Par son caractère exceptionnellement tardif, l'épisode de crue de juin 2016 a pu être isolé des événements hydrologiques hivernaux précédents, sur les " dalles de crue "; mais malheureusement, seul un échantillon sur " dalle annuelle" fut exploitable compte tenu de son homogénéité granulométrique (lit sableux récolté sur la dalle SA1-04be; fig. 3C). Dans le même ordre d'idée, les analyses granulométriques d'une carotte à visée géochimique, réalisée en 2015 dans le banc du site de Sainte-Anne (JOSAN15C3; fig. 3C), ont été utilisées à titre de comparaison; plus précisément, on a retenu une unité sableuse située vers $29 \mathrm{~cm}$ de profondeur, comparable à la récolte effectuée sur dalle. Des prélèvements sédimentaires de la charge de fond du lit de l'Orne ont également été effectués à proximité immédiate des deux bancs en juin 2017 (HP5-1 et SA1-1; fig. 3B et 3C), après une année hydrologique particulièrement calme (crue maximale inférieure à $40 \mathrm{~m}^{3} / \mathrm{s}$ en débit moyen journalier).

Toutes les mesures évoquées, hydrologiques, topographiques et sédimentologiques, ont servi de point d'appui à des calculs hydrodynamiques, pour lesquels on a essayé de minimiser l'utilisation de formules hydrauliques et sans chercher à établir une modélisation. La démarche a été la suivante :

1) Calcul du rayon hydraulique à la section HP1, près de la passerelle d'Haropré, basé sur des mesures topographiques et à l'aide de la mesure à l'A.D.C.P. effectuée le $1^{\text {er }}$ juin 2016 au droit de cette passerelle. 
2) Cette même mesure du $1^{\mathrm{er}}$ juin 2016 à $14 \mathrm{~h}$ fournit une vitesse moyenne et le débit de référence $\left(136,5 \mathrm{~m}^{3} / \mathrm{s}\right.$; fig. 3D).

3) Mesure de la pente du niveau d'eau à partir des repères de crue à HP5 et SA1. En l'absence de point de repère précis à HP1, une pente régulière $(0,0915 \%)$ a été adoptée sur tout le tronçon (fig. 3E). On notera que cette valeur de pente est proche de celle fournie par SINBIO (2013a) et par le M.N.T. de l'I.G.N. (2017) dans le fond du lit de l'Orne, sur l'ensemble de la zone de remous du barrage de Beth (environ 0,085\%), ce qui tend à montrer l'annulation de l'influence de l'ouvrage hydraulique dans le secteur concerné en période de grosse crue.

4) Calcul de la pente de la ligne de charge (Se), barrage de Beth ouvert, entre la passerelle d'Haropré et SA1. La formule de Manning - Strickler appliquée aux mesures à l'A.D.C.P. permet l'obtention d'un coefficient de rugosité K $(26,9)$, cohérent avec le milieu fluvial considéré (CEMAGREF/IRSTEA in Fort et al., 2015).

5) Identification du coefficient $\mathrm{K}$ et calcul de la vitesse moyenne au droit de la section HP1, pour le même débit de $136,5 \mathrm{~m}^{3} / \mathrm{s}$. La valeur de $\mathrm{K}$ reste logiquement proche de ce qui a été obtenu précédemment (25).

6) Détermination du coefficient de rugosité sur les sites HP5 et SA1, sur le modèle de 1'étape 5, à partir des données obtenues à HP1 et d'équations issues de précédents jaugeages réalisés sur chaque site dans diverses situations hydrologiques. Les différences morphologiques et hydrodynamiques impliquent des coefficients $\mathrm{K}$ variant entre 24,3 et 28,4 pour les quatre sections. La formule de Manning - Strickler est également ajustée au débit de référence, de manière à obtenir une vitesse moyenne à chaque section et, par voie de conséquence, pouvoir calculer et tracer la ligne de charge (fig. 3E).

7) Calcul de la puissance spécifique et de la contrainte tractrice sur chaque site, pour les sections HP6 et SA1 (fig. 6). On notera que la puissance spécifique sera fournie préférentiellement en utilisant la pente de la ligne d'eau et non celle de la ligne de charge, afin de pouvoir effectuer des comparaisons avec des données de la littérature (Schmitt et al., 2001). La contrainte tractrice, quant à elle, a été calculée de deux façons : par le rayon hydraulique et la pente de la ligne de charge d'une part, et par la puissance spécifique réelle et la vitesse moyenne. Les résultats sont très proches et seuls ceux de la première méthode de calcul seront utilisés pour caractériser la mobilisation sédimentaire. En revanche, en l'absence de mesure de vitesse effective sur les deux sites d'étude, il n'est pas apparu pertinent d'effectuer les calculs de contrainte tractrice par le biais de la vitesse de cisaillement (Petit, 1989 ; Bravard et Petit, 2002). La distinction entre résistance des particules et résistance des formes du lit ne sera pas non plus évoquée. 
8) Détermination des particules mises en mouvement dans les conditions hydrodynamiques obtenues, par deux approches : la contrainte tractrice critique et le paramètre de Shields (Bravard et Petit, 2002 ; Degoutte, 2006; Le Hir, 2008). Les résultats sont ensuite comparés aux dépôts sédimentaires effectivement constatés à l'issue du passage de la crue.

\section{Hydrodynamique de l'Orne et implication pour la mobilisation sédimentaire}

Les deux sites d'étude révèlent une puissance spécifique comparable, de l'ordre de $30 \mathrm{~W} / \mathrm{m}^{2}$ (fig. 6), ce qui fait de l'Orne un cours d'eau d'assez faible énergie hydrodynamique ; il serait tout juste capable de changements morphologiques dans cette zone amont de remous (Brookes in Wasson et al., 1998). La puissance spécifique réelle (calculée à partir de la pente de la ligne de charge) tombe même à $18 \mathrm{~W} / \mathrm{m}^{2}$ sur le site HP5.

Pour autant, les vitesses du courant (moyennes à $1,15 \mathrm{~m} / \mathrm{s}$ à HP5 et $1,39 \mathrm{~m} / \mathrm{s}$ à SA1) autorisent ablation et transport de sédiments de taille presque centimétrique lors d'une crue biennale ( $c f$. diagramme de Hjulström; Chamley, 2000). Des particules plus grosses pourraient alors être exportées en considérant les vitesses maximales dans le chenal et plus précisément les zones de gradients de vitesse les plus importantes près du fond (Dzana et Gaillard, 1996).

Les contraintes tractrices moyennes avoisinent les 15 et $22 \mathrm{~N} / \mathrm{m}^{2}$ à HP5 et SA1 respectivement (fig. 6). L'initiation de la mise en mouvement des sédiments s'effectue à partir d'une valeur de contrainte tractrice critique, dont la relation avec le diamètre des particules a fait l'objet de nombreux travaux, en contextes artificiel et naturel (Bravard et Petit, 2002 ; fig. 7A). A partir des résultats de contraintes tractrices moyennes obtenus, on peut établir une gamme de taille minimale de particules pouvant être emportées par le flux pendant la crue. Les valeurs s'échelonnent entre 15 et $81 \mathrm{~mm}$ à $\mathrm{HP} 5$ et entre 22 et $99 \mathrm{~mm}$ à SA1. Elles représentent une très grande part, voire presque la totalité de la charge de fond de l'Orne pouvant être mobilisée, d'après les analyses granulométriques réalisées sur les prélèvements HP5-1 et SA1-1 (85 et 97\% du sédiment respectivement; fig. 7B). Cette première approche par la contrainte tractrice critique peut être complétée et précisée par une seconde approche liée au paramètre de Shields.

La médiane granulométrique (D50) des deux échantillons HP5-1 et SA1-1 donne des valeurs du critère de Shields de 0,39 et 0,27 respectivement (fig. 8). Ces résultats sont bien supérieurs aux seuils de charriage proposés par A. Shields et par M. Ramette ( $\tau^{*}$ entre 0,047 et 0,06 , voire 0,138 
pour les rivières à graviers avec granulométrie étalée liée à un fond pavé ; $c f$. Degoutte, 2006). Malgré un coefficient d'uniformité (D40/D90) supérieur à 3, donc avec une granulométrie étalée (Degoutte, 2006), l'Orne à Jœuf constitue une rivière à graviers à fond non pavé, d'après les observations de terrain et comme l'indiquent les courbes granulométriques des échantillons HP5-1 et SA1-1 (fig. 7B : symétrie ou légère asymétrie positive de Folk et Ward; $c f$. Miskovsky et Debard, 2002 ; Losson et Corbonnois, 2006). Quoi qu'il en soit, il apparaît qu'une crue biennale est capable de détruire un pavage qui serait présent temporairement.

Dans un tel contexte morphosédimentaire, les valeurs de contrainte tractrice fournissent un paramètre de Shields qui rend compte d'un début de déplacement par charriage pour des particules jusqu'à $19,9 \mathrm{~mm}$ de diamètre à HP5 et $28,7 \mathrm{~mm}$ à SA1 (lorsque $\tau^{*}>0,047$ ). Cela représente respectivement à peu près l'équivalent du décile et du centile le plus grossier de chaque population granulométrique (fig. 7B), c'est-à-dire que 90 et $99 \%$ des particules ont pu bouger pendant la crue. Avec ces valeurs, on se trouve dans la fourchette inférieure des résultats de la première approche.

Pour une dimension granulométrique donnée, toute chose égale par ailleurs, l'accroissement de l'énergie de la rivière peut aboutir à un changement de mode de transport des particules, en passant du charriage à la saltation, puis à la suspension (Chamley, 2000). Le seuil de mise en suspension des particules a été évalué pour différentes valeurs du paramètre de Shields, selon les auteurs (fig. 8). Pour le cas de rivières à sable, M. Ramette propose d'élever le seuil de $\tau^{*}$ à 2,5 , valeur à partir de laquelle les formes de type rides disparaissent ( $c f$. Degoutte, 2006). La taille maximale des particules qui ont pu être mises en suspension pendant la crue du $1^{\mathrm{er}}$ juin 2016, a été estimée à partir de trois seuils proposés (tab. 1). Il s'avère que le transport en suspension de sables de dimension inférieure à $370 \mu \mathrm{m}$ à $\mathrm{HP} 5$ et $540 \mu \mathrm{m}$ à SA1 était envisageable pendant le pic de crue, d'après la classification de M. Ramette ; la valeur à SA1 pourrait atteindre 1,1 mm si l'on se base sur les travaux de R.A. Bagnold. En revanche, les résultats fondés sur le seuil de L.C. Van Rijn s'éloignent sensiblement des données sédimentaires obtenues sur le banc SA1 (fig. 9), que ce soit sur dalle de sédimentation (SA1-04be; dépôt de 2016 avec un percentile grossier C1 de $941 \mu \mathrm{m}$ ) ou par carottage (JOSAN15C3-29; unité sableuse d'un probable dépôt post-1967, voire 1988, de percentile grossier $\mathrm{C} 1 \mathrm{de} 758 \mu \mathrm{m})$.

La dalle SA1-04be en particulier, située entre 1,5 et $2 \mathrm{~m}$ au-dessus du fond du lit de l'Orne (cf. fig. 3C) et qui présente essentiellement des sables fins et moyens (fig. 5B et $9:$ médiane de $200 \mu \mathrm{m}$, taille moyenne de Folk et Ward à $130 \mu \mathrm{m}$; $c f$. Miskovsky et Debard, 2002 ; Losson et Corbonnois, 2006), a vraisemblablement recueilli des sédiments transportés en suspension, éventuellement combinée à de la saltation; on peut exclure un remaniement local des alluvions du banc ou un 
apport latéral. Cela est confirmé par des observations de dépôts superficiels de sables fins sur la ripisylve après la crue et par l'absence de particules graveleuses sur les dalles de sédimentation, ni ailleurs en surface de banc, ni dans les carottages effectués. L'unité sableuse analysée sur SA1-04be a toutefois pu être mise en place pendant plusieurs phases de crues de l'année hydrologique 20152016 (crues du 10 février et du 31 mai 2016, de même intensité que celle du 1er juin ; crue du 4 juin 2016 qui a en revanche atteint $167 \mathrm{~m}^{3} / \mathrm{s}$ ), sans que les dynamiques de remaniement sédimentaire entre ces phases ne soient déterminées.

\section{Conclusion}

On peut considérer que le rétablissement de l'écoulement naturel de l'Orne, par effacement du barrage de Beth, donnerait lieu à de l'érosion des dépôts sédimentaires actuellement présents à l'amont de la zone de remous. La puissance spécifique n'est de ce point de vue pas très révélatrice de la capacité de réajustement morphologique du cours d'eau. Ce fait a déjà été souligné par de nombreux auteurs (Petit et al., 2005 ; Petit et al., 2008 ; Corbonnois et al., 2016), en raison de la combinaison de multiples facteurs énergétiques et morphosédimentaires (Malavoi et Bravard, 2010).

A l'occasion de la crue biennale du $1^{\text {er }}$ juin 2016, équivalent localement à un débit à pleins bords, des mesures hydrologiques, topographiques et sédimentologiques ont été menées pour fiabiliser autant que possible les calculs de contrainte tractrice ; cette dernière a servi à évaluer la population granulométrique des particules qui ont été mises en mouvement. L'approche par la contrainte tractrice critique a laissé entrevoir un potentiel de mobilisation sédimentaire bien supérieur à ce que propose le diagramme de Hjulström par les vitesses (les valeurs moyennes étant considérées dans les deux cas). L'utilisation du paramètre de Shields a permis de préciser une initiation des mouvements par charriage pour des particules jusqu'à $2-3 \mathrm{~cm}$ de diamètre, soit une taille deux à trois fois plus grande que celle estimée par le biais des vitesses moyennes. Cela représente une possibilité de mise en mouvement de plus de $90 \%$ de la charge de fond lors d'une crue biennale. Le même paramètre de Shields a aussi permis de considérer les particules qui ont pu migrer en suspension pendant la crue ; le transport de sables jusqu'à $1 \mathrm{~mm}$ de diamètre par ce mode de prise en charge par le courant est attesté par l'analyse de dépôts ayant engraissé les bancs sédimentaires présents sur les sites d'étude. 
Les crues de l'Orne sont donc bien en capacité de remobiliser toute la gamme de particules limoneuses à graveleuses dans le secteur d'étude. Les analyses géochimiques, minérales et organiques, sur les sédiments limono-sableux du banc de Sainte-Anne, ont mis en lumière une contamination assez faible par rapport à d'autres dépôts situés plus en aval dans la zone de remous du barrage de Beth. Et dans le cadre du projet d'arasement de cet ouvrage, il convient de s'intéresser de plus près au secteur aval. Les bancs, étudiés par ailleurs (Kanbar, 2017 ; Kanbar et $a l ., 2017)$, présentent des caractéristiques granulométriques nettement plus faibles à proximité du barrage (taille moyenne autour de $14 \mu \mathrm{m}$, contre $37 \mu \mathrm{m}$ à Sainte-Anne) et avec un caractère cohésif fortement marqué. Cependant, des atterrissements sableux grossiers existent aussi en fond de lit (SINBIO, 2013a ; fig. 2A). Si la modélisation hydraulique qui sera réalisée dans ce secteur aval doit prendre en considération ces différences sédimentaires, elle pourra s'appuyer sur les mesures effectuées à l'amont pour établir les conditions d'ablation qui pourraient être mises en jeu en cas d'effacement du barrage.

\section{Remerciements}

Cette recherche franco-luxembourgeoise est financée par l'Agence Nationale de la Recherche et le Fonds National de la Recherche (étude MOBISED, «Modelling the remobilization of sediments and the release of associated contaminants »; ANR-14-CE01-0019 et FNR-INTER/ANR/13/45). Elle s'inscrit dans un site atelier de la ZAM (Zone Atelier Moselle, réseau e-LTER), également soutenu financièrement par l'Agence de l'Eau Rhin-Meuse.

\section{Références}

Banque HYDRO (2019a) - L'Orne à Moyeuvre-Grande. Ministère de l'écologie, du développement durable et de l'énergie [en ligne : http://hydro.eaufrance.fr/selection.php] (consulté le 9 août 2019).

Banque HYDRO (2019b) - L'Orne à Rosselange. Ministère de l'écologie, du développement durable et de l'énergie [en ligne : http://hydro.eaufrance.fr/selection.php] (consulté le 9 août 2019).

Bravard J.-P., Petit F. (2002) - Les cours d'eau. Dynamique du système fluvial. Armand Colin, Paris, 2e édition, $222 \mathrm{p}$.

Chamley H. (2000) - Bases de sédimentologie. Dunod, Paris, 2e édition, 178 p. 
Cheng F., Granata T. (2007) - Sediment transport and channel adjustments associated with dam removal: Field observations. Water Resources Research, 43, 3, W03444, 14 p.

DOI : 10.1029/2005WR004271

Corbonnois J., Bonnefond M., Chardon V., Rodrigues S., Jugé P., Cali J., Verdun J., Simonetto E., Tchekpo W., Labergerie E, Faucheux G. (2016) - Détermination des conditions de la dynamique fluviale d'une rivière aménagée de basse énergie, à partir de secteurs du Loir (bassin de la Loire aval). Géomorphologie : relief, processus, environnement, 22, 4, 345-361.

DOI : 10.4000/geomorphologie.11559

Degoutte G. (2006) - Diagnostic, aménagement et gestion des rivières. Hydraulique et morphologie fluviales appliquées. Tec \& Doc, Lavoisier, Paris, 394 p.

Dzana J.-G., Gaillard S. (1996) - Hydrodynamique et mobilité d'une boucle de méandre au stade du débit à pleins bords. L'exemple de la Seine supérieure. Géomorphologie : relief, processus, environnement, 2, 13-27.

E.P.F.L. (2009) - Orne - Fensch, d'hier à aujourd'hui... préservons l'avenir. Etablissement public foncier de Lorraine, Pont-à-Mousson, 48 p. [en ligne : http://www.epfl.fr/PDF/Fascicule_Orne.pdf] (consulté le 22 mars 2016).

Fort M., Bétard F., Arnaud-Fassetta G. (2015) - Géomorphologie dynamique et environnement. Armand Colin, collection U, 336 p.

Garcier R.J. (2007) - Rivers we can't bring ourselves to clean - historical insights into the pollution of the Moselle River (France), 1850-2000. Hydrology and Earth System Sciences, 11, 6, 1731-1745. DOI : 10.5194/hess-11-1731-2007

I.G.N. (2017) - Géoportail [en ligne : https://www.geoportail.gouv.fr/] (consulté le 4 avril 2017).

Justica P. (2015) - Etude morphodynamique de l'Orne dans le secteur de Jouf (Lorraine). Rapport de stage de Master 1, Université de Lorraine, 27 p.

Kanbar H.J. (2017) - What the Orne River tells about the former steelmaking activities: chemical and mineralogical investigations on sediments. Thèse de doctorat, Université de Lorraine et Université Libanaise, 262 p.

Kanbar H.J., Montargès-Pelletier E., Losson B., Bihannic I., Gley R., Bauer A., Villiéras F., Manceau L., El Semrani A.G., Kazpard V., Mansuy-Huault L. (2017) - Iron mineralogy as a fingerprint of former steelmaking activities in river sediments. Science of the Total Environment, 599-600, 540-553.

DOI : 10.1016/j.scitotenv.2017.04.156

Le Hir P. (2008) - Aide mémoire de dynamique sédimentaire. IFREMER, Brest, 7e édition, 74 p. 
Losson B., Corbonnois J. (2006) - Les modes de sédimentation détritique : nouvelle méthode de détermination appliquée à des remplissages endokarstiques. Geologica Belgica, 9, 3-4, 257-265.

Malavoi J.-R., Bravard J.-P. (2010) - Eléments d'hydromorphologie fluviale. ONEMA, 224 p. [en ligne : http://www.onema.fr/elements-d-hydromorphologie-fluviale] (consulté le 26 janvier 2011).

Manière L. (2013) - Mise au point d'un protocole d'évaluation de l'impact des ouvrages transversaux sur la continuité sédimentaire dans le cadre d'une approche multiscalaire. Rapport de stage de Master 2, Université Lumière Lyon 2, 78 p.

Martinois R. (2014) - Histoire d'Orne. Cercle pour la Promotion de l'Histoire de Jœuf, 25 p. [en ligne : http://cphj.free.fr/Histoire/Histoire d'Orne.pdf] (consulté le 20 juin 2014).

Melun G. (2012) - Evaluation des impacts hydromorphologiques du rétablissement de la continuité hydro-sédimentaire et écologique sur l'Yerres aval. Thèse de doctorat, Université Paris Diderot (Paris 7), $335 \mathrm{p}$.

Miskovsky J.-C., Debard E. (2002) - Granulométrie des sédiments et étude de leur fraction grossière. In: Miskovsky J.-C. (éd.) - Géologie de la préhistoire. Géopré, PU Perpignan, Association pour l'étude de l'environnement géologique de la préhistoire, Paris, 479-501.

Petit F. (1989) - Evaluation des critères de mise en mouvement et de transport de la charge de fond en milieu naturel. Bulletin de la Société Géographique de Liège, 25, 91-111.

Petit F., Hallot E., Houbrechts G., Levecq Y., Mols J., Peeters A., Van Campenhout J. (2008) La typologie et les caractéristiques hydromorphologiques des cours d'eau wallons. In : Bilan d'une décennie d'ingénierie écologique, actes du colloque «La Gestion physique des cours d'eau», Service Public de la Wallonie, Namur, octobre 2007, 7-16 [en ligne : http://hdl.handle.net/2268/40148] (consulté le 16 juillet 2016).

Petit F., Hallot E., Houbrechts G., Mols J. (2005) - Evaluation des puissances spécifiques de rivières de moyenne et de haute Belgique. Bulletin de la Société Géographique de Liège, 46, 37-50.

Perrot A. (2014) - Etude morphologique du lit mineur de l'Orne entre Jouf et le barrage de Beth à Moyeuvre-Grande (Lorraine). Rapport de stage de Licence 3, Université de Lorraine, 27 p.

Picon M. (2014) - Autour de l'Orne industrieuse: paysages industriels hérités. Mémoire de Master, Conservatoire national des Arts et Métiers, Ecole supérieure des Géomètres et Topographes, $192 \mathrm{p}$.

Schmitt L., Maire G., Humbert J. (2001) - La puissance fluviale : définition, intérêt et limites pour une typologie hydro-géomorphologique de rivières. Zeitschrift für Geomorphologie, 45, 2, 201-224.

SINBIO (2013a) - Etude pour l'aménagement du barrage de Beth sur la commune de MoyeuvreGrande. Opération d'ouverture temporaire du vannage pour abaissement maximal de la retenue en 
vue de l'inspection du fond et des berges. Bilan de l'opération. Rapport d'étude pour le Syndicat intercommunal de curage de l'Orne, septembre 2013, 18 p.

SINBIO (2013b) - Etude pour l'aménagement du barrage de Beth sur la commune de MoyeuvreGrande. Mission $1+$ mission 2. Diagnostic global, propositions de scénarios d'aménagement, bilan de l'opération d'ouverture temporaire du barrage, étude détaillée des scénarios d'aménagement. Rapport d'étude pour le Syndicat intercommunal de curage de l'Orne, décembre 2013, 75 p.

Syed A.U., Bennett J.P., Rachol C.M. (2005) - A pre-dam-removal assessment of sediment transport for four dams on the Kalamazoo River between Plainwell and Allegan, Michigan. U.S. Geological Survey Scientific Investigations Report 2004-5178, 41 p.

Wasson J.-G., Malavoi J.-R., Maridet L., Souchon Y., Paulin L. (1998) - Impacts écologiques de la chenalisation des rivières. CEMAGREF Éditions (collection «Etudes», série «Gestion des milieux aquatiques », 14), $158 \mathrm{p}$. 
Titres des figures et tableau :

Fig. 1 - L'Orne entre Jœuf et Moyeuvre-Grande : contexte géomorphologique du site d'étude. 1. Isohypse (équidistance : $40 \mathrm{~m}) ; 2$. Point coté $(\mathrm{m}) ; 3$. Cours d'eau, avec zone de remous des barrages en foncé ; 4. Substratum à dominante calcaire (Bajocien et Aalénien); 5. Substratum à dominante marneuse (Toarcien) ; 6. Versant de concavité de méandre encaissé ; 7. Versant $>20 \mathrm{~m}$ de commandement, en fond de vallée ; 8. Glacis de lobe convexe de méandre encaissé ; 9. Vallon en $\mathrm{V} ; 10$. Vallon en berceau ; 11. Lit majeur faisant encore office de plaine d'inondation ; 12. Barrage au fil de l'eau et station hydrométrique ; 13. Zone urbanisée ; 14. Zone bouleversée par les activités industrielles.

Fig. 1 - The Orne River between Jouf and Moyeuvre-Grande: geomorphological setting of the study area. 1. Isohypse (contour interval: $40 \mathrm{~m}$ ); 2. Spot height (in m); 3. River, with backwater of the dams in dark; 4. Mainly calcareous bedrock (Bajocian and Aalenian); 5. Mainly marly bedrock (Toarcian); 6. Concave slope of enclosed meander; 7. Valley side with dislevelment $>20 \mathrm{~m}$, in valley bottom; 8. Slope of convex lobe of enclosed meander; 9. V-shaped vale; 10. Dale; 11. Still functional flood plain; 12. Run-of-the-river dam and hydrometric station; 13. Urban area; 14. Disrupted area after industrial activities.

Fig. 2 - Deux aperçus du secteur d'étude. A : Le barrage de Beth lors de son ouverture en été 2013 (10 juillet 2013). B : Partie aval du banc alluvial SA1 sur le site Sainte-Anne (21 mars 2016).

Fig. 2 - Two views of the study area. A: The Beth Dam when it was opened during the summer of 2013 (July 10 ${ }^{\text {th }}$ 2013). B: The part downstream of the SAl bar at the Sainte-Anne site (March $21^{\text {st }}$ 2016).

Fig. 3 - Contexte morphosédimentaire et hydrodynamique de l'Orne dans la zone de remous amont du barrage de Beth. A : Cartographie morphosédimentaire du lit mineur, d'après Justica (2015, modifié). B : Topographie du site HP5 en septembre 2016, avec représentation du niveau de crue du $1^{\text {er }}$ juin 2016. C : Topographie du site SA1 en septembre 2016 (complétée en rive gauche avec celle de mai 2015), avec représentation du niveau de crue du $1^{\text {er }}$ juin 2016. D : Hydrogramme à la station JOAB pendant l'épisode de crues de fin mai - début juin 2016. E : Profil longitudinal entre les sites HP5 et SA1; cas d'une pente d'eau régulière de $0,09 \%$ pendant la crue du $1^{\text {er }}$ juin 2016. 1. Cours d'eau ; 2. Seuil ; 3. Berge douce, stable; 4. Berge sapée ; 5. Berge enrochée ; 6. Berge bétonnée; 7. Blocs épars ; 8. Banc d'alluvions fines ; 9. Rive boisée ; 10. Rive à végétation arbustive ou arborée clairsemée; 11. Rive à végétation herbacée; 12. Emprise végétale sur 
l'écoulement en étiage (arbre, grosse branche, broussaille); 13. Encoche d'érosion localisée ; 14. Source ; 15. Ponton ; 16. Buse ; 17. Passerelle ; 18. Section de mesure ; 19. Point topographique ; 20. Pied et branche(s) de saule; 21. Dalle de sédimentation; 22. Prélèvement sédimentaire ; 23. Carottage ; 24. Ligne de charge ; 25. Ligne d'eau ; 26. Fond du lit.

Fig. 3 - Morphosedimentary and hydrodynamic context of the Orne River in the upstream backwater zone of the Beth Dam. A: Morphosedimentary mapping of the riverbed, modified from Justica (2015). B: Topography of the HP5 site in September 2016, with representation of the flood

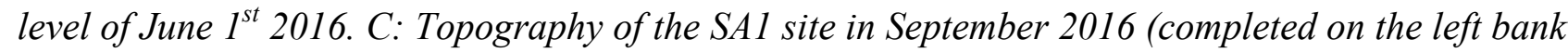
with that of May 2015), with representation of the flood level of June $1^{\text {st }}$ 2016. D: Hydrograph at the JOAB station during the flood episode late May-early June 2016. E: Longitudinal profile between HP5 and SA1 sites; case of a regular water slope of 0.09\% during the flood of June $1^{\text {st }}$ 2016. 1. River; 2. Riffle; 3. Gentle and stable bank; 4. Undercut bank; 5. Enrocked bank; 6. Concreted bank; 7. Scattered blocks; 8. Fine alluvial bar; 9. Wooded bank; 10. Bank with shrubs or sparse trees; 11. Bank with herbaceous vegetation; 12. Plant influence on the flow in low water level (tree, large branch, scrub); 13. Localized erosion notch; 14. Spring; 15. Pontoon; 16. Duct; 17. Footbridge; 18. Measurement section; 19. Topographic point; 20. Foot and branch(es) of willow; 21. Sedimentation slab; 22. Sedimentary sampling; 23. Core sampling; 24. Energy line; 25. Water level; 26. Bottom of the riverbed.

Fig. 4 - Aperçu de la crue du $1^{\text {er }}$ juin 2016 au niveau de la passerelle d'Haropré (section HP1 ; cf. fig. 3A). Le bas de l'escalier se situe environ 1,3 m sous la surface de l'eau.

Fig. 4 - View of the flood of June $1^{\text {st }} 2016$ at Haropré footbridge (section HP1; see fig. 3A). The bottom of the stairs is approximately $1.3 \mathrm{~m}$ below the surface of the water.

Fig. 5 - Dépôt sédimentaire sur la dalle SA1-04be, entre septembre 2015 et septembre 2016. A : Matérialisation de la dalle enfouie sous l'accumulation sédimentaire annuelle. B : Coupe dans le dépôt ; l'unité sableuse analysée ( $c f$. fig. 9) se limite à la partie supérieure du dépôt.

Fig. 5 - Sedimentary deposit on the slab SA1-04be, between September 2015 and September 2016. A: Marking of the slab buried under the annual sedimentary deposition. B: Section in the deposit; the sand unit analyzed (see fig. 9) is limited to the upper part of the deposit).

Fig. 6 - Données hydrodynamiques des sites HP5 (section HP6) et SA1 (section SA1). $\omega r$ : puissance spécifique réelle calculée avec la pente de la ligne de charge $\mathrm{Se}, \omega$ : puissance spécifique calculée avec la pente de la ligne d'eau S, $\rho$ : masse volumique de l'eau $\left(1000 \mathrm{~kg} / \mathrm{m}^{3}\right), \mathrm{g}$ : 
accélération de la pesanteur $\left(9,81 \mathrm{~m} / \mathrm{s}^{2}\right), \mathrm{Qb}$ : débit à pleins bords, $\mathrm{w}$ : largeur du lit mineur à pleins bords, $\tau$ : contrainte tractrice, $\mathrm{Rh}$ : rayon hydraulique, $\mathrm{Vm}$ : vitesse moyenne.

Fig. 6 - Hydrodynamic data for the HP5 (HP6 section) and SA1 (SA1 section) sites. $\omega r$ : true unit stream power calculated with the energy grade line Se, $\omega$ : unit stream power calculated with the hydraulic gradient $S, \rho$ : density of water $\left(1000 \mathrm{~kg} / \mathrm{m}^{3}\right), \mathrm{g}$ : gravitational acceleration $\left(9,81 \mathrm{~m} / \mathrm{s}^{2}\right)$, Qb: bankfull discharge, w: bankfull width, $\tau$ : shear stress, Rh: hydraulic radius, Vm: mean velocity.

Fig. 7 - Caractéristiques hydrosédimentaires pour les sites HP5 et SA1 : approche par la contrainte tractrice critique $(\tau \mathbf{c})$. A : Diamètre des particules en fonction de la contrainte tractrice critique, d'après différents auteurs (in Bravard et Petit, 2002). B : Granulométrie totale de la charge de fond à HP5 et SA1 (D10 = décile représentant les $10 \%$ de particules les plus grossières ; D50 = médiane). 1. Diamètre entre 15 et $81 \mathrm{~mm}$ maximum à HP5, soit $85 \%$ minimum des particules de fond de lit ( $c f$. graphe B - 3) ; 2. Diamètre entre 22 et 99 mm maximum à SA1, soit $97 \%$ minimum des particules de fond de lit ( $c f$. graphe B - 4). 3. Évaluation graphique du pourcentage de particules à $15 \mathrm{~mm}$ pour HP5-1. 4. Évaluation graphique du pourcentage de particules à $22 \mathrm{~mm}$ pour SA1-1.

Fig. 7 - Hydrosedimentary characteristics for HP5 and SA1 sites: critical shear stress approach

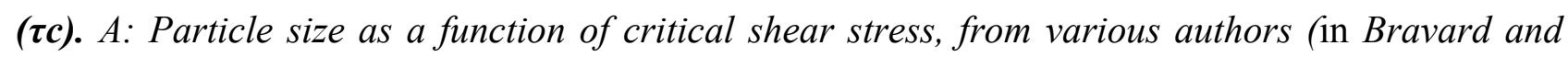
Petit, 2002). B: Total grain-size distribution of the bedload at HP5 and SA1 (D10 = decile representing the $10 \%$ of the coarsest particles; D50 = median). 1. Size between 15 and $81 \mathrm{~mm}$ maximum at HP5, i.e. 85\% minimum of the bedload (see graph B - 3); 2. Size between 22 and $99 \mathrm{~mm}$ maximum at SA1, i.e. 97\% minimum of the bedload (see graph B - 4). 3. Graphical evaluation of the percentage of particles at $15 \mathrm{~mm}$ for HP5-1; 4. Graphical evaluation of the percentage of particles at $22 \mathrm{~mm}$ for $S A 1-1$.

Fig. 8 - Caractéristiques hydrosédimentaires pour les sites HP5 et SA1 : approche par le paramètre de Shields $\left(\tau^{*}\right)$. Paramètre adimensionnel de Shields $\tau^{*}$ en fonction du diamètre adimensionnel des particules D*, d'après Van Rijn in Le Hir (2008), modifié. $\gamma$ séd: poids volumique du sédiment, produit de la masse volumique et de l'accélération de la pesanteur $\left(\sim 26 \mathrm{kN} / \mathrm{m}^{3}\right)$, yeau : poids volumique de l'eau $\left(\sim 9,8 \mathrm{kN} / \mathrm{m}^{3}\right), \mathrm{D}$ : diamètre des particules (en $\left.\mathrm{m}\right), \mathrm{g}$ : accélération de la pesanteur $\left(9,81 \mathrm{~m} / \mathrm{s}^{2}\right), v$ : viscosité cinématique de l'eau $\left(1,12.10^{-6} \mathrm{~m}^{2} / \mathrm{s}\right.$ à $\left.15^{\circ} \mathrm{C}\right)$.

Fig. 8-Hydrosedimentary characteristics for HP5 and SA1 sites: Shields parameter approach $(\tau *)$. Dimensionless Shields parameter $\tau^{*}$ as a function of the dimensionless diameter of the particles $D^{*}$, from Van Rijn in Le Hir (2008), modified. $\gamma$ séd : specific weight of sediment, product of density and gravitational acceleration $\left(\sim 26 \mathrm{kN} / \mathrm{m}^{3}\right)$, yeau : specific weight of water $\left(\sim 9,8 \mathrm{kN} / \mathrm{m}^{3}\right)$, 
$D$ : size of particles (in $\mathrm{m}), \mathrm{g}$ : gravitational acceleration $\left(9,81 \mathrm{~m} / \mathrm{s}^{2}\right), v:$ kinematic viscosity of water $\left(1,12.10-6 \mathrm{~m}^{2} / \mathrm{s}\right.$ at $\left.15^{\circ} \mathrm{C}\right)$.

Tab. 1 - Dimension maximale des particules mises en suspension, selon différentes valeurs du paramètre de Shields, proposées par divers auteurs.

Tab. 1 - Maximum size of suspended particles, according to different Shields parameter values, proposed by various authors.

Fig. 9 - Granulométrie totale de deux dépôts sableux du banc SA1. C1 = percentile représentant le premier pourcent de particules les plus grossières ; D50 = médiane ; Tm = taille moyenne de Folk et Ward ( $c f$. Miskovsky et Debard, 2002 ; Losson et Corbonnois, 2006).

Fig. 9- Total grain-size distribution of two sand deposits of SA1 bar. C1 = percentile representing the first percent of coarsest particles; D50 = median; Tm = mean size of Folk and Ward (see Miskovsky and Debard, 2002; Losson and Corbonnois, 2006). 


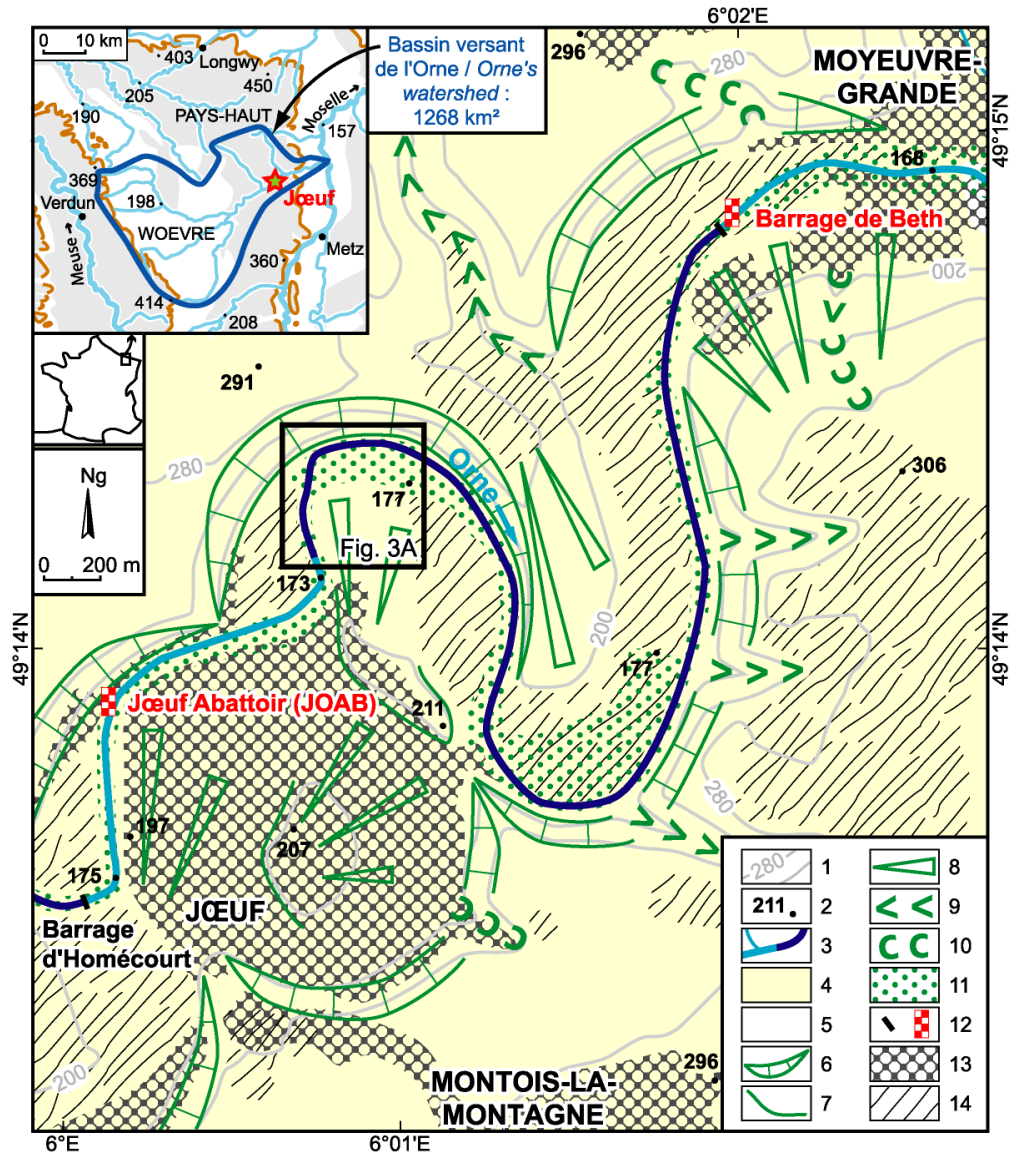


(D)

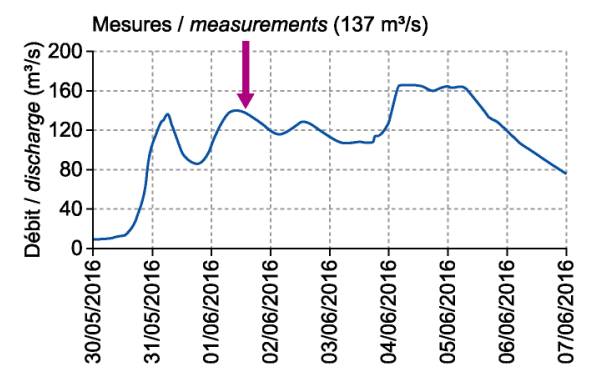

(E)

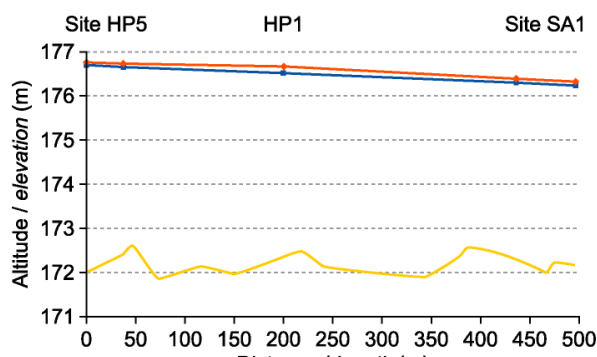

Distance / length $(\mathrm{m})$
$-24-25-26$

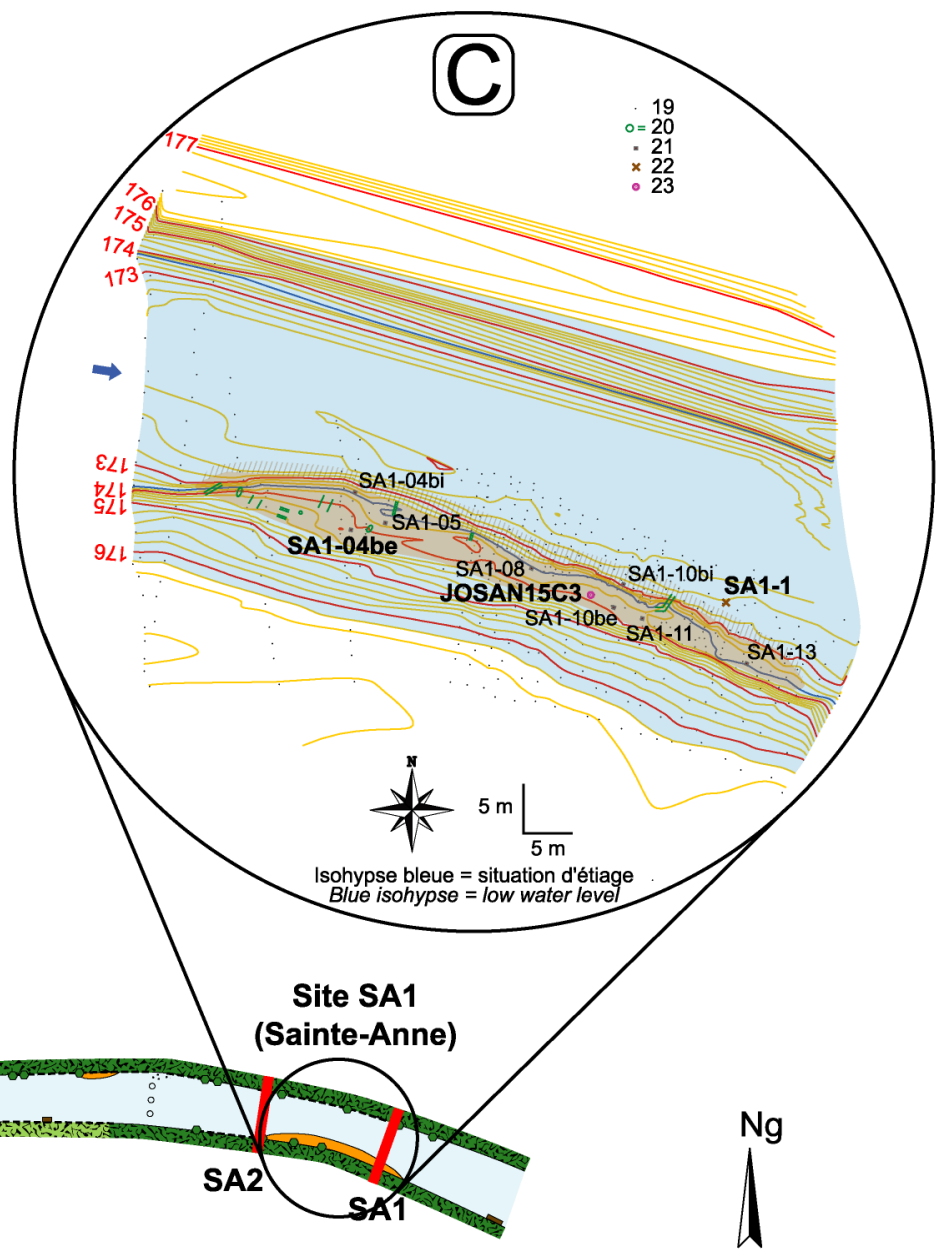

0

$100 \mathrm{~m}$

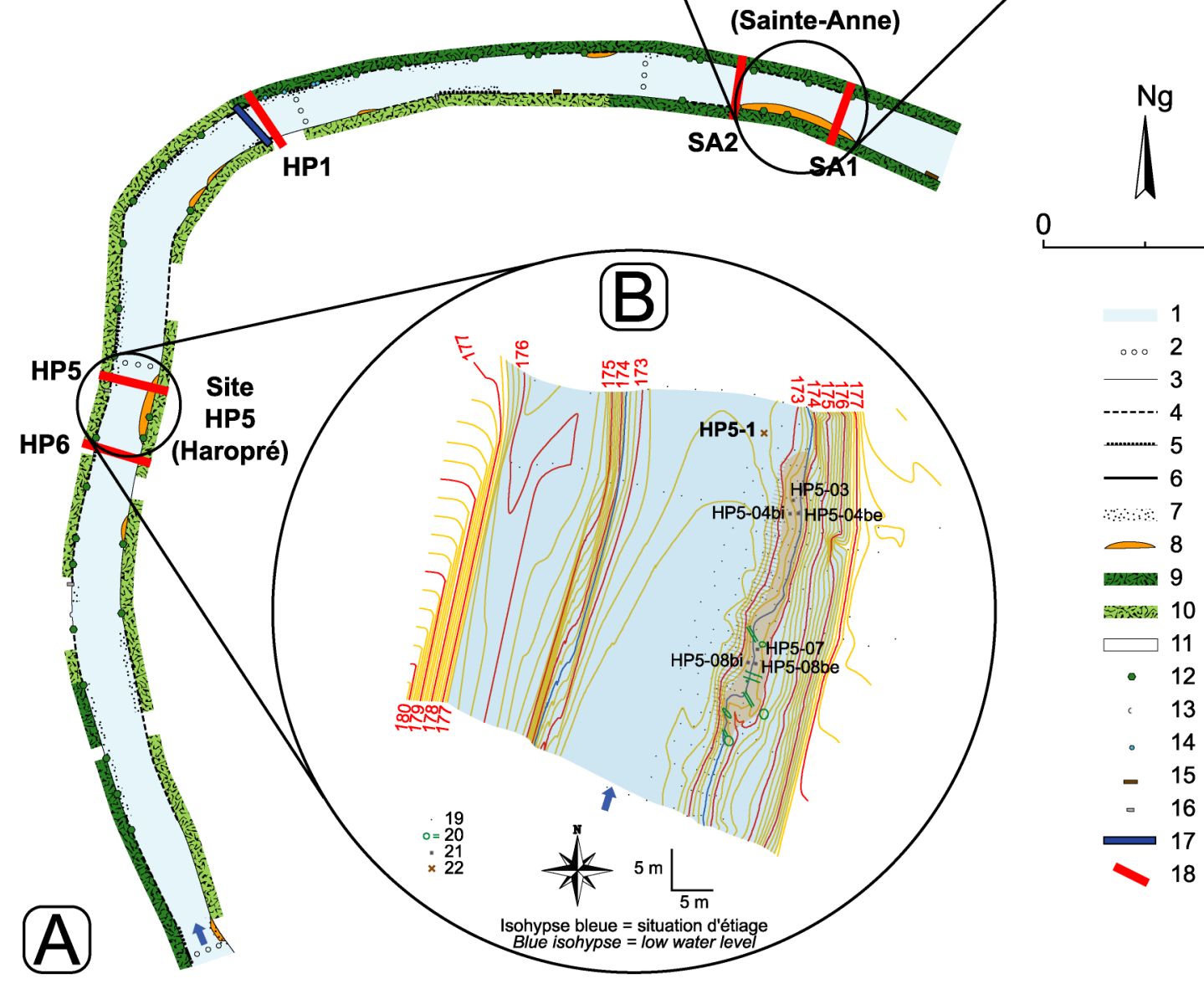



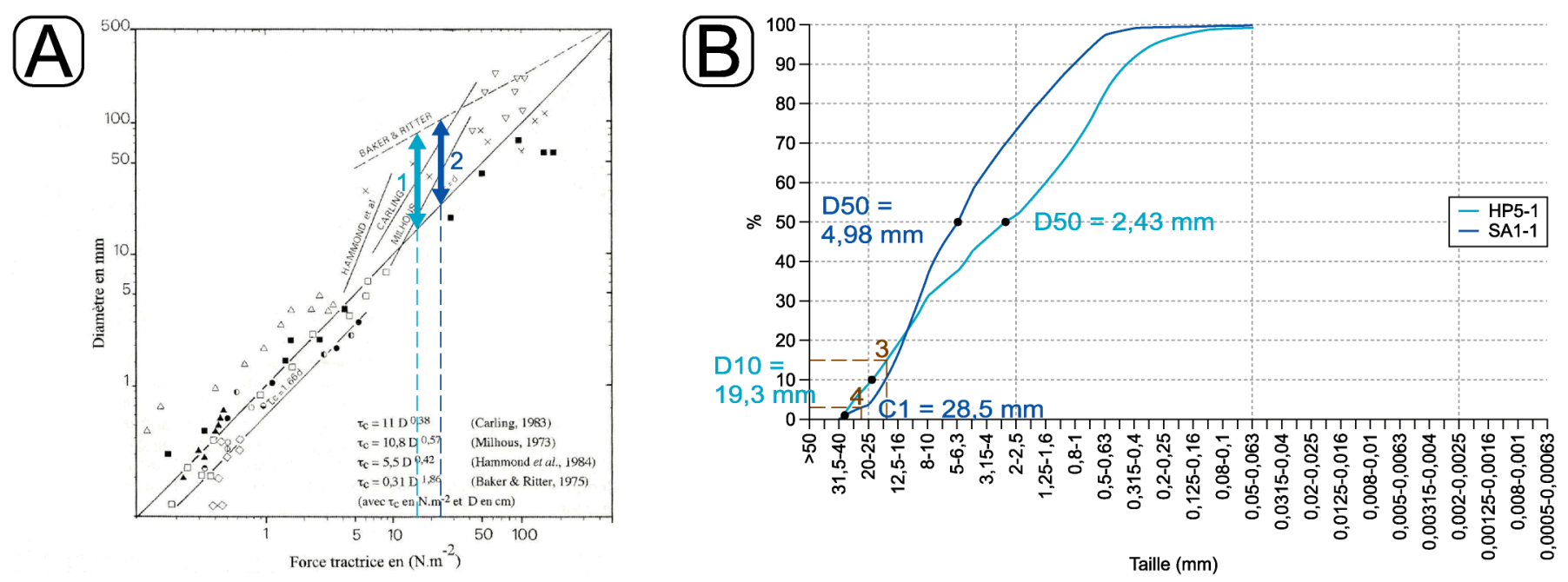


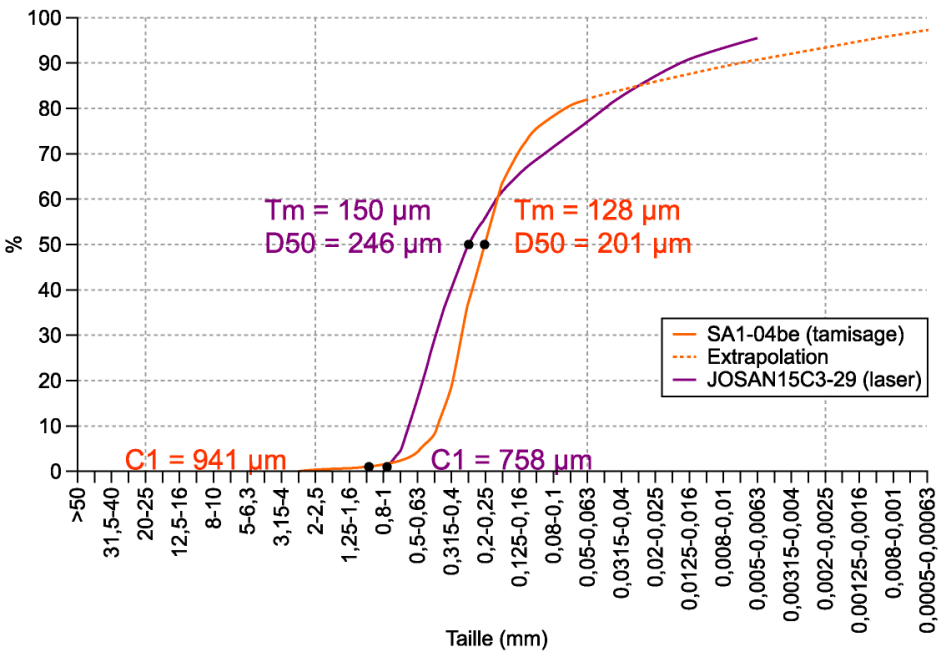

